\title{
$\left[{ }^{18} \mathrm{~F}\right]$-Fluciclovine PET discrimination of recurrent intracranial metastatic disease from radiation necrosis
}

\author{
Ephraim E. Parent ${ }^{1 \dagger}$, Dhruv Patel ${ }^{2 \dagger}$, Jonathon A. Nye ${ }^{2}$, Zhuo Li ${ }^{3}$, Jeffrey J. Olson ${ }^{4}$, David M. Schuster ${ }^{2}$
} and Mark M. Goodman ${ }^{2 *}$ (D)

\begin{abstract}
Background: Stereotactic radiosurgery (SRS) is often the primary treatment modality for patients with intracranial metastatic disease. Despite advances in magnetic resonance imaging, including use of perfusion and diffusion sequences and molecular imaging, distinguishing radiation necrosis from progressive tumor remains a diagnostic and clinical challenge. We investigated the sensitivity and specificity of ${ }^{18} \mathrm{~F}$-fluciclovine PET to accurately distinguish radiation necrosis from recurrent intracranial metastatic disease in patients who had previously undergone SRS.
\end{abstract}

Methods: Fluciclovine PET imaging was performed in 8 patients with a total of 15 lesions that had previously undergone SRS and had subsequent MRI and clinical features suspicious for recurrent disease. The SUVmax of each lesion and the contralateral normal brain parenchyma were summated and evaluated at four different time points (5 min, $10 \mathrm{~min}, 30 \mathrm{~min}$, and $55 \mathrm{~min}$ ). Lesions were characterized as either recurrent disease (11 of 15 lesions) or radiation necrosis (4 of 15 lesions) and confirmed with histopathological correlation (7 lesions) or through serial MRI studies (8 lesions).

Results: Time activity curve analysis found statistically greater radiotracer accumulation for all lesions, including radiation necrosis, when compared to contralateral normal brain. While the mean and median SUV max $_{\text {for recurrent }}$ disease were statistically greater than those of radiation necrosis at all time points, the difference was more significant at the earlier time points ( $p=0.004$ at 5 min-0.025 at 55 min). Using a SUV $\max$ threshold of $\geq 1.3$, fluciclovine PET demonstrated a 100\% accuracy in distinguishing recurrent disease from radiation necrosis up to 30 min after injection and an accuracy of $87 \%$ (sensitivity $=0.91$, specificity $=0.75$ ) at the last time point of $55 \mathrm{~min}$. However, tumor-to-background ratios $\left(\mathrm{TBR}_{\max }\right)$ were not significantly different between recurrent disease and radiation necrosis at any time point due to variable levels of fluciclovine uptake in the background brain parenchyma.

Conclusions: Fluciclovine PET may play an important role in distinguishing active intracranial metastatic lesions from radiation necrosis in patients previously treated with SRS but needs to be validated in larger studies.

Keywords: ${ }^{18} \mathrm{~F}$-fluciclovine, Brain metastasis, Radiation necrosis, Amino acid, PET

*Correspondence: mgoodma@emory.edu

${ }^{\dagger}$ Ephraim E. Parent and Dhruv Patel contributed equally to the work

${ }^{2}$ Department of Radiology and Imaging Sciences, Emory University School of Medicine, 1841 Clifton Rd. NE, 2nd Floor, Atlanta, GA 30329, USA

Full list of author information is available at the end of the article

\section{Background}

Intracranial brain metastases from an extracranial primary lesion are seen in $24-45 \%$ of patients with known melanoma, lung, breast, and renal primary cancers [1]. Imaging plays a crucial role in management of metastatic brain lesions, and conventional magnetic resonance imaging (MRI) has been the standard of care 
for detection, treatment planning, and post-treatment evaluation of brain metastasis [2]. In patients previously treated with SRS, both tumor recurrence and radiation necrosis lead to clinical deterioration and can have a similar appearance on both anatomic imaging with MRI and computed tomography (CT), as well as with metabolic scans such as magnetic resonance spectroscopy and positron emission tomography (PET) $[3,4]$.

2-deoxy-2 $\left[{ }^{18} \mathrm{~F}\right]$ fluoro-D-glucose (FDG) PET has been shown to have limited ability to accurately identify recurrent intracranial metastatic disease after radiation therapy $[5,6]$. Many tumors exhibit upregulated amino acid transporter expression and, as a result, have been shown to concentrate radiolabeled amino acids $[7,8]$. Because normal brain parenchyma does not concentrate significant amino acid radiotracer, radiolabeled amino acids can provide a high signal-to-noise ratio and improve evaluation in the post-radiation setting where radiation necrosis may be confused with recurrent disease on MRI and clinical evaluation $[9,10]$.

Amino acid PET including L-methyl-[C-11]-methionine ([C-11]-MET) PET and 6-[F-18]-fluoro-L-dopa ([F-18]-FDOPA) PET has been shown to help differentiate active malignancy from radiation necrosis for intracranial metastasis [11-13]. Anti-1-amino-3-[F18]-fluorocyclobutane-1-carboxylic acid, anti-3-[F-18] FACBC (fluciclovine), is a synthetic amino acid that has been shown to have high uptake in tumors and was approved by the US Food and Drug Administration (FDA) in May 2016 for the indication of biochemical evidence of recurrent prostate cancer [14]. It has also been shown to have increased uptake in brain glioma for which it has been granted orphan drug status [15-18]. Recently, it has also been reported to differentiate between highgrade and low-grade gliomas [15]. The purpose of this study was to investigate the ability of fluciclovine to discriminate recurrent intracranial metastatic disease from radiation necrosis in patients previously treated with SRS. We hypothesized that using simple semiquantitative PET parameters, such as maximum standardized uptake value (SUVmax), fluciclovine would be able distinguish recurrent metastatic disease from radiation necrosis in patients with known intracranial metastatic disease.

\section{Methods}

\section{Patient population}

\section{Subject recruitment}

Patients with biopsy-proven primary brain glioma or intracranial metastatic disease were recruited from $09 / 17 / 2000$ to $11 / 18 / 2002$. All procedures performed in studies involving human participants were in accordance with the ethical standards of the institutional and/or national research committee and with the 1964
Declaration of Helsinki and its later amendments or comparable ethical standards. The recruitment protocol was approved by the Institutional Review Board (IRB) and complied with the Health Insurance Portability and Accountability Act (HIPAA). The data were collected as part of a phase I trial for whole-body imaging under the IRB title 'Imaging Analysis of Amino Acid Metabolism In Intracranial Tumors Using PET and ${ }^{18} \mathrm{~F}$-FACBC (IRB\#00101652)' and submitted on $1 / 27 / 2002$. This study is not listed on clinicaltrials.gov because when it was submitted, clinicaltrails.gov was only requiring $\mathrm{NIH}$-funded projects be listed. After completion of a related fluciclovine PET study to evaluate gliomas [15], we have now analyzed the original dataset to focus on patients with suspected recurrent intracranial metastatic disease. Informed consent was obtained from all individual participants included in the study. The radiotracer was administered under FDA Investigational New Drug (IND) 72,437 and was synthesized either via automated synthesis [19] or the FASTlab Cassette System (GE Healthcare). Safety monitoring during the drug infusion was performed, and no adverse events were recorded.

For this analysis, patients were sub-selected under the inclusion criteria of intracranial metastatic disease previously treated with SRS, with a mean time between completion of SRS and fluciclovine PET of 10.5 months (range 1.2-18.3 months). In total, 25 patients were recruited and received at least one fluciclovine PET study post-histologic confirmation as required by the IRB. No patients had undergone fluciclovine PET studies prior to SRS for comparison. Patients were followed up with either excisional biopsy/partial resection or serial brain MRI examinations for up to 2 years or death. Patients that were lost to follow-up were not included in this analysis. The reference standard for disease progression was histologic proof when possible or progressive enlargement on serial MRI. A negative biopsy or stability/improvement on serial MRI without interval therapy was considered consistent with radiation necrosis. It is well known that histological analysis of radiation necrosis often finds three distinct patterns: viable tumor, radiation necrosis and most often a mix of necrosis and viable cells [20], with this final combination possibly confounding image interpretation. Unfortunately, due to the delay between tissue sampling and this study, the original tissue samples were not available for review and the negative or positive biopsy results are based on the original pathology report. Patients were excluded from analysis if they received SRS within 5 weeks prior to fluciclovine PET to exclude confounding inflammation from acute radiotherapy. This resulted in a total of 8 patients with 15 lesions included in this study (Table 1 ). 
Table 1 Patient demographics

\begin{tabular}{lll}
\hline Age & $\mathbf{5 2}$ years (39-86) & \\
\hline Gender & 4 Male & 4 Female \\
Primary tumor & Patients & Lesions \\
Lung & $4(50 \%)$ & $5(33 \%)$ \\
Renal & $1(13 \%)$ & $4(27 \%)$ \\
Breast & $2(25 \%)$ & $3(20 \%)$ \\
Colon & $1(13 \%)$ & $3(20 \%)$ \\
\hline
\end{tabular}

\section{Image acquisition}

All studies were collected on an ECAT 921 dedicated PET scanner in 3D mode consisting of 24 crystal rings spanning a field of view $16.2 \mathrm{~cm}$ resulting in 47 reconstructed image planes. Fluciclovine scans were acquired over $65 \mathrm{~min}$ in dynamic mode and started concurrently with injection of $357 \pm 24 \mathrm{MBq}$ of activity. The data were collected in sonogram mode and binned into four time points $(2 \times 5 \mathrm{~min}, 1 \times 20 \mathrm{~min}$, and $1 \times 25 \mathrm{~min}$ ). PET emission data were corrected for attenuation, randoms, and scatter and reconstructed with a filtered backprojection algorithm and Hanning filter $(0.4 \times$ Nyquist frequency) giving an in-plane resolution of $7.8 \mathrm{~mm}$ full width at half maximum (FWHM) and axial resolution of $6.2 \mathrm{~mm}$ FWHM. Data were transferred to a MIM workstation (MIM Software, $\mathrm{OH}$ ) for further analysis.

\section{Selection of regions of interest (ROIs)}

A board-certified radiologist using the Absolute Threshold Contouring Tool (MIM Software, OH, USA) drew ROIs over the tumors and background ROIs (i.e., contralateral brain and venous confluence) for all time points. Fluciclovine PET images were co-registered to T1 post-contrast MRI and fluid-attenuated inversion recovery (FLAIR) sequences. Tumor ROIs were defined by creating a spherical PET ROI to include the volume of tissue demonstrating contrast enhancement corresponding to known intracranial metastatic deposit. Within this PET ROI, the voxels with peak activity were used to derive a tumor maximum standardized uptake value $\left(S U V_{\max }\right)$. A $15-\mathrm{mm}$ spherical ROI was placed over the contralateral normal brain, including both gray and white matter as appropriate, to obtain a normal maximum standardized uptake value $\left(\mathrm{SUV}_{\text {max nor- }}\right.$ mal). Careful consideration when drawing ROIs over the tumor was used to exclude blood pool or adjacent choroid plexus which could falsely contribute to artifactually elevated SUV values.

\section{Semiquantitative PET metrics}

$\mathrm{SUV}_{\max }$ for each lesion and contralateral normal parenchyma was recorded at all time points. Tumorto-background ratios for each lesion were calculated as $\mathrm{TBR}_{\max }=\left(\mathrm{SUV}_{\max }\right.$ tumor $) /\left(\mathrm{SUV}_{\text {max normal }}\right)$ at all imaged time points.

Estimating threshold values for classification of recurrent metastatic disease versus radiation necrosis.

The optimal threshold for differentiating radiation necrosis from recurrent disease utilizing tumor $\mathrm{SUV}_{\max }$ was calculated using a receiver operator characteristic curve (ROC) for each lesion $\mathrm{SUV}_{\max }$ measurements from 5 to $55 \mathrm{~min}$ post-injection. Sensitivity and specificity for identifying radiation necrosis are reported based on the optimal threshold. A similar approach using ROC curves was applied to each $\mathrm{TBR}_{\max }$ dataset to distinguish recurrent metastatic disease versus radiation necrosis.

\section{Statistical analysis}

For each time point, mean SUVmax and the standard deviation of SUVmax were calculated for normal brain parenchyma and brain lesions. Statistical significance between malignant lesions and radiation necrosis was determined using Wilcoxon rank sum test. All tests were two-sided with alpha level set at 0.05 for statistical significance. R3.6.1 was used for analysis.

\section{Results}

Subject demographics

Eight patients (4 male and 4 female) with intracranial malignancies previously treated with SRS and a mean age of 52 years (range $39 y-86 y$ ) were included per the inclusion criteria (Table 1).

One patient had 4 lesions, another had 3 lesions, two patients had 2 lesions each, and the remaining four had 1 lesion each, resulting in a total of 15 distinct lesions being independently evaluated. Lung cancer was the most common primary malignancy with the highest number of patients (4 of 8 ) and metastatic lesions (5/15). Other primary malignancies included renal (1 patient/4 lesions), breast ( 2 patients $/ 3$ lesions), and colon ( 1 patient $/ 3$ lesions). All patients completed the fluciclovine PET scan after standard-of-care MRI demonstrated an enhancing lesion in an area previously treated with SRS, with mean time between completion of SRS and fluciclovine PET of 10.5 months (range 1.2 to 18.3 months). Histological confirmation via stereotactic biopsy/excisional biopsy was obtained for 7 lesions with the remaining 8 lesions classified with either progressive enhancement (recurrent tumor) or stable/decreasing enhancement (radiation necrosis) on subsequent standard-of-care MRI 
examinations. Based on their subsequent pathological and/or MRI findings, 11 lesions met criteria for recurrent disease and 4 lesions met criteria for radiation necrosis. Histological verification included 5 of 11 lesions (45\%) with recurrent disease and 2 of 4 patients (50\%) with radiation necrosis. PET imaging was performed an average of 11.1 months (range 1 months -18 months) after completion of SRS.

\section{Semiquantitative PET metrics and threshold values}

ROC analysis was performed for each lesion and compared to contralateral normal brain parenchyma at four different time points: $5 \mathrm{~min}, 10 \mathrm{~min}, 30 \mathrm{~min}$, and 55 min (Table 2). Each lesion, including those with radiation necrosis, demonstrated statistically greater radiotracer accumulation compared to normal brain parenchyma at each time point. The mean and median $\mathrm{SUV}_{\max }$ for recurrent disease were similarly statistically greater than those of radiation necrosis at all four time points, and greater at the earlier time points $(\mathrm{p}=0.004$ at $5 \mathrm{~min}-0.025$ at $55 \mathrm{~min}$ ). Retrospective analysis provided an optimum $S U V_{\max }$ threshold of $\geq 1.3$ to distinguish recurrent disease from radiation necrosis. Using this threshold, fluciclovine PET demonstrated a 100\% accuracy at the 5,10 , and $30 \mathrm{~min}$ time points and an accuracy of $87 \%$ at the $55 \mathrm{~min}$ time point (sensitivity $=0.91$, specificity $=0.75$ ).

In an attempt to normalize differences in physiologic vascular flow, the $\mathrm{SUV}_{\max }$ of each lesion was normalized to the contralateral brain, $\mathrm{TBR}_{\max }=\left(\mathrm{SUV}_{\max }\right.$ tumor $) /\left(\mathrm{SUV}_{\text {max_normal }}\right)$, and these values were compared between the two groups. However, $\mathrm{TBR}_{\max }$ was not significantly different between recurrent disease and radiation necrosis at any time point in this analysis due to variable levels of fluciclovine uptake in the background brain parenchyma (Figs. 1, 2).

One patient with low fluciclovine uptake demonstrated progressive increase in enhancement on MRI at 2 and 4 months after fluciclovine PET and subsequently underwent surgical resection without intervening chemotherapy or radiation therapy, and final pathology was consistent with radiation necrosis. For the remainder of the lesions that underwent surgical resection, both pathology and follow-up MRI were consistent with each other.

Table 2 SUVmax values for recurrent disease, radiation necrosis, and normal brain

\begin{tabular}{|c|c|c|c|c|}
\hline & Recurrent disease $(\mathrm{N}=11)$ & Tumor necrosis $(\mathrm{N}=4)$ & Background $(\mathrm{N}=15)$ & $p$ value \\
\hline 5 m Lesion & & & & 0.004 \\
\hline Mean (SD) & $1.9(0.6)$ & $0.8(0.1)$ & $0.6(0.2)$ & \\
\hline Median (range) & $2.0(1.1,3.1)$ & $0.8(0.7,1.0)$ & $0.6(0.4,0.9)$ & \\
\hline 5 min $T B R_{\max }$ & & & & 0.121 \\
\hline Mean (SD) & $3.0(1.3)$ & $1.8(0.2)$ & & \\
\hline Median (range) & $2.6(1.3,5.3)$ & $1.8(1.6,2.2)$ & & \\
\hline 10 min Lesion & & & & 0.033 \\
\hline Mean (SD) & $2.3(1.2)$ & $0.9(0.2)$ & $0.6(0.2)$ & \\
\hline Median (range) & $2.0(1.4,5.4)$ & $0.9(0.7,1.0)$ & $0.6(0.4,1.0)$ & \\
\hline 10 min TBR $\max$ & & & & 0.129 \\
\hline Mean (SD) & $3.4(1.8)$ & $1.9(0.5)$ & & \\
\hline Median (range) & $3.1(1.5,7.8)$ & $1.8(1.5,2.6)$ & & \\
\hline 30 min Lesion & & & & 0.042 \\
\hline Mean (SD) & $2.3(1.1)$ & $1.1(0.2)$ & $2.0(1.1)$ & \\
\hline Median (range) & $2.2(1.4,5.3)$ & $1.1(0.8,1.3)$ & $1.9(0.8,5.3)$ & \\
\hline $30 \min ^{T B R_{\max }}$ & & & & 0.178 \\
\hline Mean (SD) & $3.6(1.9)$ & $2.2(0.6)$ & & \\
\hline Median (range) & $3.2(1.4,7.7)$ & $2.3(1.5,2.8)$ & & \\
\hline 55 min Lesion & & & & 0.025 \\
\hline Mean (SD) & $2.3(0.9)$ & $1.1(0.3)$ & $0.7(0.2)$ & \\
\hline Median (range) & $2.3(1.3,4.4)$ & $1.1(0.8,1.4)$ & $0.6(0.2,1.2)$ & \\
\hline 55 min TBR $\max$ & & & & 0.304 \\
\hline Mean (SD) & $3.3(1.5)$ & $2.5(0.9)$ & & \\
\hline Median (range) & $3.2(1.4,5.7)$ & $2.5(1.4,3.5)$ & & \\
\hline
\end{tabular}

* P value for each time point between mean SUVmax of malignant metastatic lesion and radiation necrosis 


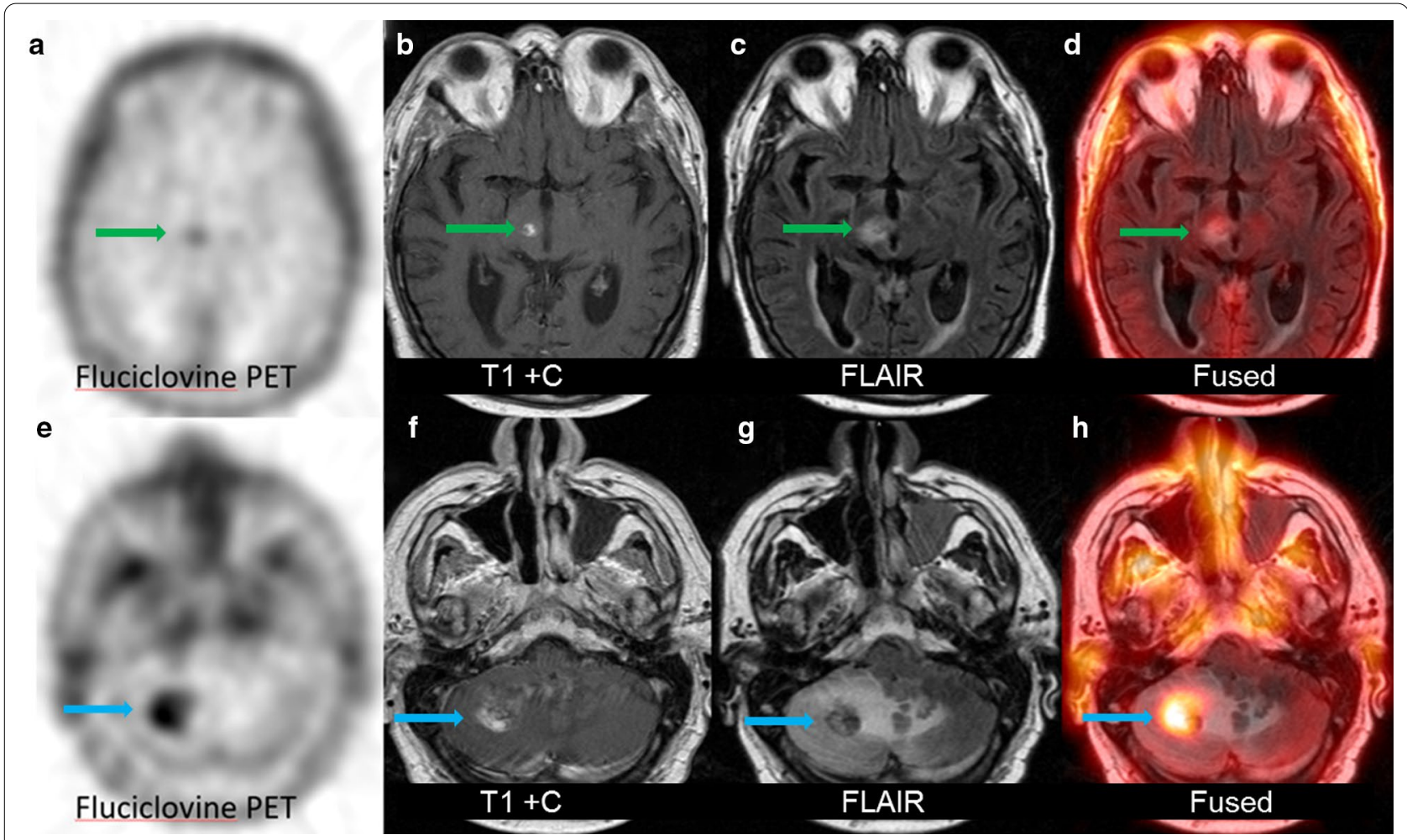

Fig. 1 A 54-year-old patient with metastatic renal cell carcinoma and prior stereotactic radiosurgery. Follow-up MRI demonstrated progressively enhancing brain lesions suspicious for recurrent disease. Top panel demonstrates that a right thalamic lesion (green arrow) had low fluciclovine uptake (SUV $V_{\max }$ of 1.0) as seen on transaxial PET (a), corresponding T1 + contrast (b), focal FLAIR hyperintensity (c), and fused FLAIR and PET (d). This lesion did not increase in size on follow-up MRI and was considered consistent with radiation necrosis. A right cerebellar lesion (blue arrow) in

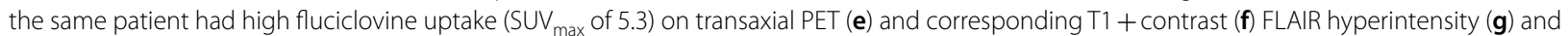
fused FLAIR and PET (h). The right cerebellar lesion was found to be recurrent metastatic disease upon resection

\section{Discussion}

Metastatic brain tumors are the most common brain tumor in adults, and the frequency of brain metastasis is increasing with up to 200,000 new cases every year [2]. External beam radiation therapy, in particular SRS, is considered part of first-line therapy for intracranial metastases [21]. The efficacy of SRS in patients with intracranial metastases has been shown to have control rates of 70-90\% [22]. One of the most common problems of SRS for both primary brain gliomas and intracranial metastases is correctly identifying progressive reactive changes from radiation injury. Early true progression is difficult to distinguish from reactive changes (pseudoprogression) in the short term and irreversible injury (radiation necrosis) at latter time points [23]. Radiation necrosis is difficult to distinguish from tumor recurrence by both clinical presentation and imaging studies and can be seen in up to $25 \%$ of patients after SRS [24]. Both recurrent tumor and radiation necrosis demonstrate increased FLAIR signal and disruption of the blood brain barrier resulting in contrast enhancement $[3,13]$. The ability to accurately identify true progression from therapy-related changes is critical as it enables appropriate therapeutic intervention. Even with MRI techniques such as perfusion [25] and spectroscopy [26], differentiation between radiation necrosis and active metastatic brain lesion is difficult and brain biopsy remains the gold standard [3].

FDG, while widely used, has discordant results in its ability to differentiate recurrent brain metastasis from radiation necrosis, possible due to different thresholds used in each study and elevated background brain parenchymal uptake [27]. Amino acid PET agents such as [F-18]-fluroethyltyrosine ([F-18]-FET) and [C-11]-MET $[28,29]$ have been used with some success as a means to differentiate progressive metastatic disease from radiation necrosis. [F-18]-FET TBR values have been shown to accurately identify recurrent metastases with metastatic uptake being significantly higher than that of radiation necrosis [30]. Additionally, dynamic FET PET imaging has been shown to improve accuracy in distinguishing recurrent disease with characteristic time-activity curves [31]. None of these most commonly used amino acid PET radiopharmaceuticals used for intracranial metastatic 


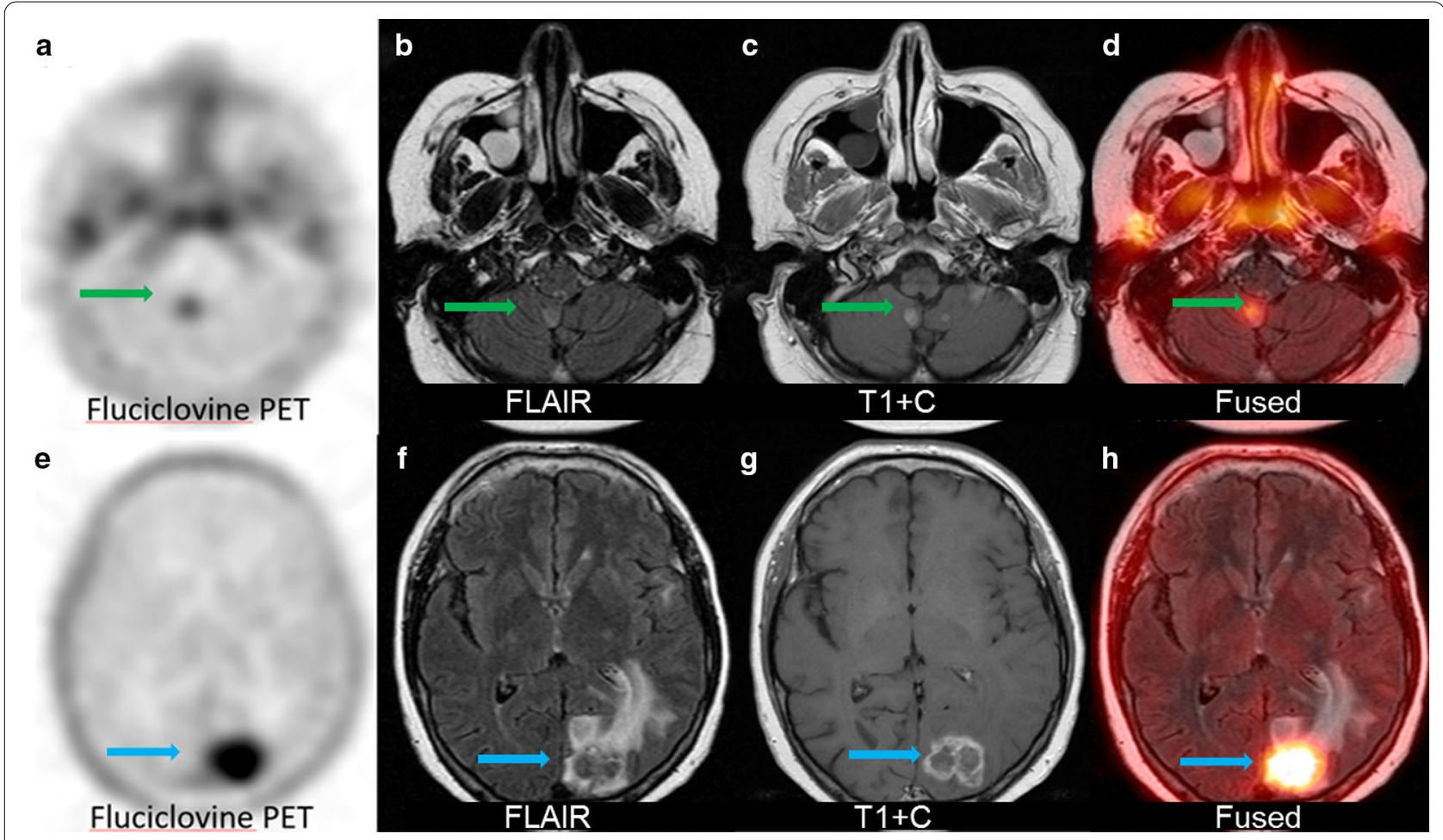

Fig. 2 A 43-year-old patient with metastatic colon cancer with prior stereotactic radiosurgery with follow-up MRI demonstrating multiple enhancing brain lesions suspicious for recurrent disease. Top panel demonstrating a right cerebellar lesion (green arrow) with low fluciclovine

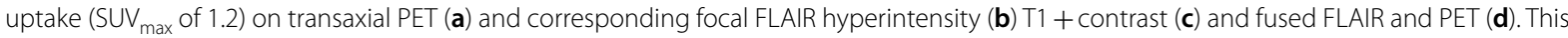
lesion did not increase in size on follow-up MRI and was consistent with radiation necrosis. A left occipital lesion (green arrow) in the same patient had high fluciclovine uptake (SUV $\max$ of 2.5) on transaxial PET (e), hyperintense FLAIR (f), T1 + contrast enhancement (g), and fused FLAIR and PET (h). The left occipital lesion was found to be recurrent metastatic disease upon resection

evaluation are yet FDA-approved and thus have limited application in research studies in the USA. Fluciclovine, on the other hand, is FDA-approved for evaluation of biochemically recurrent prostate cancer and has orphan drug status for evaluation of brain gliomas. Several other extra-prostatic malignancies including breast [32], renal [33], colon and lung (unpublished personal experience) have also been shown to have increased fluciclovine uptake. Our goal in this study was to evaluate the ability of fluciclovine to distinguish progressive metastatic lesions from radiation necrosis.

In this small sample set, all lesions, including both recurrent disease and radiation necrosis, demonstrated progressive post-contrast enhancement on prior standard-of-care MRI studies. There was overall good correlation between follow-up MRI findings and pathology results when available. It should be noted that there was a single lesion that was initially suggestive of recurrent disease on short-term follow-up with progressive increase in size and enhancement on subsequent follow-up MRI at 2 and 4 months. Conversely, there was low fluciclovine uptake in this lesion $\left(\mathrm{SUV}_{\max }\right.$ of 0.8 at 5 min increasing to
1.3 at $55 \mathrm{~min}$ ) suggestive of radiation necrosis, and radiation necrosis was confirmed upon surgical resection and final pathology.

It is important to note that fluciclovine uptake in the recurrent disease was relatively stable over the $55 \mathrm{~min}$ of imaging (Fig. 3). Conversely, fluciclovine PET uptake in radiation necrosis showed mild progressively increased uptake for the duration of the uptake scan resulting in lower accuracy at the $55 \mathrm{~min}$ time point. These observations suggest that there may be differing time-activity curves between lesions with recurrent disease and radiation necrosis which may further help distinguish them from each other, although further investigation is needed (see supplemental material). Moreover, it appears that optimal timing of image acquisition to distinguish radiation necrosis from recurrent disease for fluciclovine is at early time points (up to $30 \mathrm{~min}$ ) as progressive fluciclovine uptake in radiation necrosis lesions at later times points may confound discrimination. It is important to note that although fluciclovine uptake in radiation necrosis was lower compared to that of recurrent disease, it remained greater compared to contralateral normal brain 


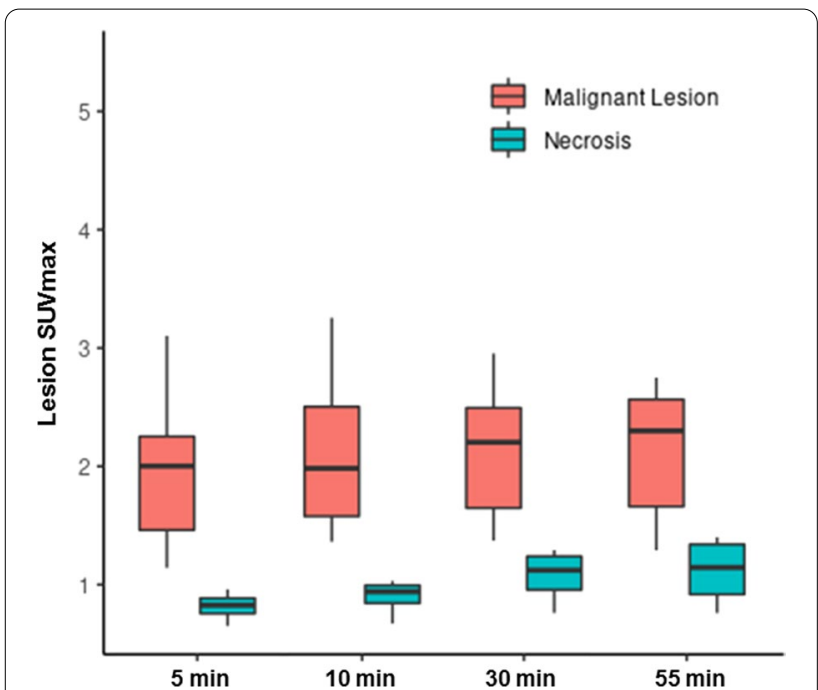

Fig. 3 Box plot diagram of SUVmax values of recurrent disease and radiation necrosis

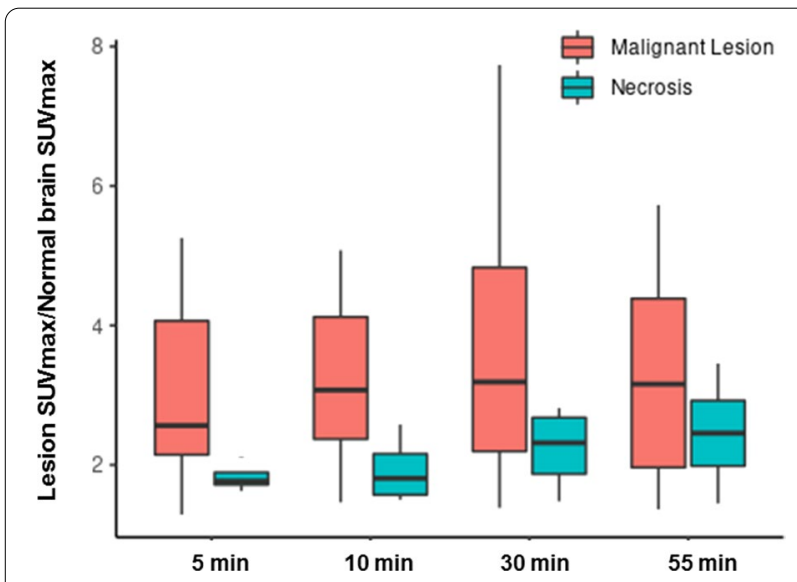

Fig. 4 Box plot diagram of $\mathrm{TBR}_{\max }=\left(S \mathrm{SV}_{\max }\right.$ tumor $) /\left(\mathrm{SUV}_{\max \text { normal }}\right)$ of recurrent disease and radiation necrosis

parenchyma. This is possibly due to fluciclovine accumulation in inflammatory processes which while less than with FDG is still present [34]. Lastly, the overlap of fluciclovine uptake also likely reflects the heterogeneity of the treated lesions with coexistent viable tumor and radiation-related changes which are typically seen on histological examination [35] (Fig. 4).

There are several limitations to our study. First is the small patient population in both the recurrent disease and radiation necrosis groups, with a total of 8 patients having 15 lesions. Of these lesions, 11 met criteria for recurrent disease and only 4 were in radiation necrosis. Despite such a small number of patients and lesions, we were able to achieve statistical significance in fluciclovine uptake between two groups with an optimal $\mathrm{SUV}_{\max }$ threshold of $\geq 1$.3. However, it is important to note that this threshold determination is considered preliminary at best due to the small sample size. As far as we can determine, there are no other published studies using fluciclovine PET as a metric to distinguish radiation necrosis from recurrent intracranial metastatic disease and thus meta-analysis against our results is not possible. A larger fluciclovine PET-MRI study is currently accruing patients at our institution building upon these preliminary data and comparing more complex PET parameters with advanced MRI techniques to distinguish radiation necrosis from recurrent disease. Secondly, pathological confirmation was not available for all of the patients and lesions were categorized based on follow-up MRI findings which is not ideal. However, in all instances in which histological verification was available, fluciclovine findings were consistent with pathology (Fig. 2), even when MRI suggested otherwise. An addition limitation is that this study included intra-cranial metastatic disease from four different primaries and no patients had fluciclovine PET prior to SRS to demonstrate fluciclovine uptake in areas of viable disease. Moreover, this study was not powered for evaluation of intracranial metastasis from any one individual primary malignancy. Finally, it is not known if there is an optimal temporal point after radiation therapy to discriminate between the two etiologies.

As mentioned previously, further investigation with larger datasets is needed to confirm these preliminary findings and to further establish optimal PET imaging parameters. Specific questions that are being evaluated include optimal timing of fluciclovine PET for evaluation of brain metastasis in post-radiation patients and evaluation of more complete fluciclovine PET parameters (e.g., SUVmean, SUVpeak, TBR $\left.\max _{\text {mean }}\right)$ and comparison with advanced MRI techniques (e.g., spectroscopy and perfusion). The observed difference in background brain fluciclovine uptake between patients with recurrent disease and radiation necrosis is unable to be adequately explained and is believed to be an artifact from the small sample size and will be further evaluated on a planned study with a larger sample size. If fluciclovine PET is found to have a high accuracy in distinguishing recurrent disease from radiation necrosis, this may help guide biopsy and obviate the current need for serial MRI evaluation after treatment, saving both time and money.

\section{Conclusions}

Accurate discrimination between recurrent intracranial metastatic disease and radiation necrosis remains a radiographic and clinical dilemma in patients that have previously undergone SRS. Visual and semiquantitative analysis of fluciclovine PET is able to correctly 
identify radiation necrosis from recurrent disease and background brain parenchyma. The simple semiquantitative metric of $\mathrm{SUV}_{\max }$ afforded a threshold of $\geq 1.3$ to discriminate between recurrent disease and radiation necrosis. The $\mathrm{SUV}_{\max }$ difference between radiation necrosis and recurrent disease was more pronounced at the earlier time points as radiation necrosis was found to slowly increase over time, whereas the fluciclovine uptake of recurrent disease remained relatively flat over 55 min after injection. While these results need to be evaluated in a larger sample size, fluciclovine PET may play an important role in distinguishing metabolically active intracranial metastatic lesions from radiation necrosis in patients previously treated with SRS.

\section{Supplementary information}

Supplementary information accompanies this paper at https://doi. org/10.1186/s13550-020-00739-6.

Additional file 1. Time Activity Curve analysis of recurrent disease and radiation necrosis.

\section{Abbreviations}

FDG: 2-Deoxy-2[ $\left[{ }^{18} \mathrm{~F}\right]$ fluoro-D-glucose; [C-11]-MET: L-methyl-[C-11]-methionine; [F-18] FDOPA: 6-[F-18]-fluoro-L-dopa; FDA: US Food and Drug Administration; IRB: Institutional Review Board; HIPAA: Health Insurance Portability and Accountability Act; IND: Investigational New Drug; FWHM: Full width at half maximum; FLAIR: Fluid-attenuated inversion recovery; [F-18]-FET: [F-18]-fluroethyltyrosine; TBR: Tumor-to-background ratio.

\section{Acknowledgements}

We thank Ronald Crowe RPh, BCNP, and the Emory Center for Systems Imaging Radiopharmacy, as well as Angie Williams, RN, for assistance on this work.

\section{Authors' contributions}

EEP and DP performed the data analysis and wrote the manuscript. JN helped with the data analysis and image construction. ZL did statistical analysis. JJO performed the tissue biopsies. DMS and MMG designed the study. All authors read and approved the final manuscript.

\section{Funding}

Funding was provided by Nihon Medi-physics Co., Ltd.

\section{Availability of data and materials}

Please contact author for data requests.

\section{Ethics approval and consent to participate}

All procedures performed in studies involving human participants were in accordance with the ethical standards of the institutional and/or national research committee and with the 1964 Declaration of Helsinki and its later amendments or comparable ethical standards. The recruitment protocol was approved by the Institutional Review Board (IRB) and complied with the Health Insurance Portability and Accountability Act (HIPPA). Informed consent was obtained from all individual participants included in the study.

\section{Consent for publication}

Informed consent was obtained from all individual participants included in the study.

\section{Competing interests}

The authors have participated in sponsored research involving 18F-fluciclovine, among other radiotracers. Emory University and Dr. Mark Goodman are eligible to receive royalties for $18 \mathrm{~F}$-fluciclovine. The other authors declare that they have no competing interests.

\section{Author details}

${ }^{1}$ Department of Radiology, Mayo Clinic, Jacksonville, USA. ${ }^{2}$ Department of Radiology and Imaging Sciences, Emory University School of Medicine, 1841 Clifton Rd. NE, 2nd Floor, Atlanta, GA 30329, USA. ${ }^{3}$ Department of Statistics, Mayo Clinic, Jacksonville, USA. ${ }^{4}$ Department of Neurosurgery, Emory University School of Medicine, Atlanta, GA, USA.

Received: 21 August 2020 Accepted: 24 November 2020

Published online: 07 December 2020

\section{References}

1. Barnholtz-Sloan JS, Yu C, Sloan AE, Vengoechea J, Wang M, Dignam $J$, et al. A nomogram for individualized estimation of survival among patients with brain metastasis. Neuro Oncol. 2012;14:910-8. https://doi. org/10.1093/neuonc/nos087.

2. Ruzevick J, Kleinberg L, Rigamonti D. Imaging changes following stereotactic radiosurgery for metastatic intracranial tumors: differentiating pseudoprogression from tumor progression and its effect on clinical practice. Neurosurg Rev. 2014;37:193-201. https://doi.org/10.1007/s1014 3-013-0504-8.

3. Chuang M-T, Liu Y-S, Tsai Y-S, Chen Y-C, Wang C-K. Differentiating radiation-induced necrosis from recurrent brain tumor using MR perfusion and spectroscopy: a meta-analysis. PLoS ONE. 2016;11:e0141438. https:// doi.org/10.1371/journal.pone.0141438.

4. Galldiks N, Law I, Pope WB, Arbizu J, Langen K-J. The use of amino acid PET and conventional MRI for monitoring of brain tumor therapy. NeuroImage Clin. 2017;13:386-94.

5. Zhuang H, Pourdehnad M, Lambright ES, Yamamoto AJ, Lanuti M, Li P, et al. Dual time point ${ }^{18} \mathrm{~F}$-FDG PET imaging for differentiating malignant from inflammatory processes. J Nucl Med. 2001;42:1412-7.

6. Chao ST, Suh JH, Raja S, Lee S-Y, Barnett G. The sensitivity and specificity of FDG PET in distinguishing recurrent brain tumor from radionecrosis in patients treated with stereotactic radiosurgery. Int J Cancer. 2001;96:1917. https://doi.org/10.1002/ijc.1016.

7. Huang C, McConathy J. Radiolabeled amino acids for oncologic imaging. J Nucl Med. 2013;54:1007-10. https://doi.org/10.2967/jnumed.112.11310 0 .

8. Chaofeng $\mathrm{H}$, Jonathan M. Fluorine-18 labeled amino acids for oncologic imaging with positron emission tomography. Curr Top Med Chem. 2013;13:871-91. https://doi.org/10.2174/1568026611313080002.

9. McParland BJ, Wall A, Johansson S, Sørensen J. The clinical safety, biodistribution and internal radiation dosimetry of $\left[{ }^{18} \mathrm{~F}\right]$ fluciclovine in healthy adult volunteers. Eur J Nucl Med Mol Imaging. 2013;40:1256-64. https:// doi.org/10.1007/s00259-013-2403-1.

10. Nye JA, Schuster DM, Yu W, Camp VM, Goodman MM, Votaw JR. Biodistribution and radiation dosimetry of the synthetic nonmetabolized amino acid analogue anti- ${ }^{18}$ F-FACBC in humans. J Nucl Med. 2007;48:1017-20. https://doi.org/10.2967/jnumed.107.040097.

11. Terakawa Y, Tsuyuguchi N, Iwai Y, Yamanaka K, Higashiyama S, Takami T, et al. Diagnostic accuracy of 11C-methionine PET for differentiation of recurrent brain tumors from radiation necrosis after radiotherapy. J Nucl Med. 2008;49:694-9. https://doi.org/10.2967/jnumed.107.048082.

12. Tsuyuguchi N, Sunada I, Iwai Y, Yamanaka K, Tanaka K, Takami T, et al. Methionine positron emission tomography of recurrent metastatic brain tumor and radiation necrosis after stereotactic radiosurgery: is a differential diagnosis possible? J Neurosurg. 2003;98:1056-64. https://doi. org/10.3171/jns.2003.98.5.1056.

13. Lizarraga KJ, Allen-Auerbach M, Czernin J, DeSalles AAF, Yong WH, Phelps $\mathrm{ME}$, et al. ${ }^{18} \mathrm{~F}$-FDOPA PET for differentiating recurrent or progressive brain metastatic tumors from late or delayed radiation injury after radiation treatment. J Nucl Med. 2014;55:30-6. https://doi.org/10.2967/jnume d.113.121418.

14. Parent EE, Schuster DM. Update on ${ }^{18} \mathrm{~F}$-Fluciclovine PET for prostate cancer imaging. J Nucl Med. 2018;59:733-9. https://doi.org/10.2967/jnume d.117.204032 
15. Parent EE, Benayoun M, Ibeanu I, Olson JJ, Hadjipanayis CG, Brat DJ, et al. $\left[{ }^{18} \mathrm{~F}\right]$ Fluciclovine PET discrimination between high- and low-grade gliomas. EJNMMI Res. 2018;8:67. https://doi.org/10.1186/s13550-018-0415-3.

16. Sasajima T, Ono T, Shimada N, Doi Y, Oka S, Kanagawa M, et al. Trans1-amino-3-18F-fluorocyclobutanecarboxylic acid (anti-18F-FACBC) is a feasible alternative to $11 \mathrm{C}$-methyl-L-methionine and magnetic resonance imaging for monitoring treatment response in gliomas. Nucl Med Biol. 2013;40:808-15. https://doi.org/10.1016/j.nucmedbio.2013.04.007.

17. Wakabayashi T, luchi T, Tsuyuguchi N, Nishikawa R, Arakawa Y, Sasayama T, et al. Diagnostic performance and safety of positron emission tomography using ${ }^{18} \mathrm{~F}$-Fluciclovine in patients with clinically suspected high- or low-grade gliomas: a multicenter phase Ilb trial. Asia Ocean J Nucl Med Biol. 2017;5:10-21. https://doi.org/10.22038/aojnmb.2016.7869.

18. Kondo A, Ishii H, Aoki S, Suzuki M, Nagasawa H, Kubota K, et al. Phase lla clinical study of $\left[{ }^{18} \mathrm{~F}\right]$ fluciclovine: efficacy and safety of a new PET tracer for brain tumors. Ann Nucl Med. 2016;30:608-18. https://doi.org/10.1007/ s12149-016-1102-y.

19. McConathy J, Voll RJ, Yu W, Crowe RJ, Goodman MM. Improved synthesis of anti-[ $\left[{ }^{18} \mathrm{~F}\right] \mathrm{FACBC}$ : improved preparation of labeling precursor and automated radiosynthesis. Appl Radiat Isot. 2003;58:657-66. https://doi. org/10.1016/s0969-8043(03)00029-0.

20. Miyatake S, Nonoguchi N, Furuse M, Yoritsune E, Miyata T, Kawabata S, et al. Pathophysiology, diagnosis, and treatment of radiation necrosis in the brain. Neurol Med Chir (Tokyo). 2015;55(Suppl 1):50-9.

21. Weller M, van den Bent M, Tonn JC, Stupp R, Preusser M, Cohen-JonathanMoyal E, et al. European Association for Neuro-Oncology (EANO) guideline on the diagnosis and treatment of adult astrocytic and oligodendroglial gliomas. Lancet Oncol. 2017;18:e315-29. https://doi.org/10.1016/ S1470-2045(17)30194-8.

22. Kocher M, Soffietti R, Abacioglu U, Villa S, Fauchon F, Baumert BG, et al. Adjuvant whole-brain radiotherapy versus observation after radiosurgery or surgical resection of one to three cerebral metastases: results of the EORTC 22952-26001 study. J Clin Oncol. 2011;29:134-41. https://doi. org/10.1200/JCO.2010.30.1655.

23. Hygino da Cruz LC, Jr., Rodriguez I, Domingues RC, Gasparetto EL, Sorensen AG, . Pseudoprogression and pseudoresponse: imaging challenges in the assessment of posttreatment glioma. AJNR Am J Neuroradiol. 2011;32:1978-85. https://doi.org/10.3174/ajnr.A2397.

24. Minniti G, Clarke E, Lanzetta G, Osti MF, Trasimeni G, Bozzao A, et al. Stereotactic radiosurgery for brain metastases: analysis of outcome and risk of brain radionecrosis. Radiat Oncol. 2011;6:48. https://doi. org/10.1186/1748-717X-6-48.

25. Barajas RF Jr, Chang JS, Segal MR, Parsa AT, McDermott MW, Berger MS, et al. Differentiation of recurrent glioblastoma multiforme from radiation necrosis after external beam radiation therapy with dynamic susceptibility-weighted contrast-enhanced perfusion MR imaging. Radiology. 2009;253:486-96. https://doi.org/10.1148/radiol.2532090007.

26. Bette S, Huber T, Gempt J, Boeckh-Behrens T, Wiestler B, KehI V, et al. Local fractional anisotropy is reduced in areas with tumor recurrence in glioblastoma. Radiology. 2017;283:499-507. https://doi.org/10.1148/radio I.2016152832.
27. Li H, Deng L, Bai HX, Sun J, Cao Y, Tao Y, et al. Diagnostic accuracy of amino acid and FDG-PET in differentiating brain metastasis recurrence from radionecrosis after radiotherapy: a systematic review and metaanalysis. AJNR Am J Neuroradiol. 2018;39:280-8. https://doi.org/10.3174/ ajnr.A5472.

28. Tomura N, Kokubun M, Saginoya T, Mizuno Y, Kikuchi Y. Differentiation between treatment-induced necrosis and recurrent tumors in patients with metastatic brain tumors: comparison among ${ }^{11} \mathrm{C}$-methionine-PET, FDG-PET, MR permeability imaging, and MRI-ADC-preliminary results. AJNR Am J Neuroradiol. 2017;38:1520-7. https://doi.org/10.3174/ajnr. A5252.

29. Yomo S, Oguchi K. Prospective study of ${ }^{11} \mathrm{C}$-methionine PET for distinguishing between recurrent brain metastases and radiation necrosis: limitations of diagnostic accuracy and long-term results of salvage treatment. BMC Cancer. 2017;17:713. https://doi.org/10.1186/s1288 5-017-3702-x.

30. Lohmann P, Stoffels G, Ceccon G, Rapp M, Sabel M, Filss CP, et al. Radiation injury vs. recurrent brain metastasis: combining textural feature radiomics analysis and standard parameters may increase ${ }^{18} \mathrm{~F}$-FET PET accuracy without dynamic scans. Eur Radiol. 2017;27:2916-27. https:// doi.org/10.1007/s00330-016-4638-2.

31. Ceccon G, Lohmann P, Stoffels G, Judov N, Filss CP, Rapp M, et al. Dynamic O-(2-18F-fluoroethyl)-L-tyrosine positron emission tomography differentiates brain metastasis recurrence from radiation injury after radiotherapy. Neuro Oncol. 2017;19:281-8. https://doi.org/10.1093/neuonc/now149.

32. Ulaner GA, Goldman DA, Corben A, Lyashchenko SK, Gonen M, Lewis JS, et al. Prospective clinical trial of ${ }^{18} \mathrm{~F}$-fluciclovine PET/CT for determining the response to neoadjuvant therapy in invasive ductal and invasive lobular breast cancers. J Nucl Med. 2017;58:1037-42. https://doi. org/10.2967/jnumed.116.183335.

33. Schuster DM, Nye JA, Nieh PT, Votaw JR, Halkar RK, Issa MM, et al. Initial experience with the radiotracer anti-1-amino-3-[18F]Fluorocyclobutane1-carboxylic acid (anti-[18F]FACBC) with PET in renal carcinoma. Mol Imaging Biol. 2009;11:434-8. https://doi.org/10.1007/s11307-009-0220-5.

34. Oka S, Okudaira H, Ono M, Schuster DM, Goodman MM, Kawai K, et al. Differences in transport mechanisms of trans-1-amino-3-[ $\left.{ }^{18} \mathrm{~F}\right]$ fluorocyclobutanecarboxylic acid in inflammation, prostate cancer, and glioma cells: comparison with L-[methyl- $\left.{ }^{11} \mathrm{C}\right]$ methionine and 2-deoxy2-[18 F]fluoro-D-glucose. Mol Imaging Biol. 2014;16:322-9. https://doi. org/10.1007/s11307-013-0693-0.

35. Chernov MF, Ono Y, Abe K, Usukura M, Hayashi M, Izawa M, et al. Differentiation of tumor progression and radiation-induced effects after intracranial radiosurgery. Acta Neurochir Suppl. 2013;116:193-210. https ://doi.org/10.1007/978-3-7091-1376-9_29.

\section{Publisher's Note}

Springer Nature remains neutral with regard to jurisdictional claims in published maps and institutional affiliations.

\section{Submit your manuscript to a SpringerOpen ${ }^{\odot}$ journal and benefit from:}

- Convenient online submission

- Rigorous peer review

- Open access: articles freely available online

- High visibility within the field

- Retaining the copyright to your article

Submit your next manuscript at $\boldsymbol{\nabla}$ springeropen.com 